\title{
Nucleon-nucleon potential in finite nuclei
}

\author{
U. T. Yakhshiev, ${ }^{1,2}$ Ulf-G. Meißner, ${ }^{3,4}$ A. Wirzba, ${ }^{3,4}$ A. M. Rakhimov, ${ }^{5}$ and M. M. Musakhanov ${ }^{2}$ \\ ${ }^{1}$ Department of Physics and Nuclear Physics \& Radiation Technology Institute (NuRI), Pusan National \\ University, 609-735 Busan, Republic of Korea \\ ${ }^{2}$ Theoretical Physics Department, National University of Uzbekistan, Tashkent-174, Uzbekistan \\ ${ }^{3}$ Helmholtz Institut für Strahlen- und Kernphysik (Theorie), Universität Bonn, Nußallee 14-16, D-53115 Bonn, Germany \\ ${ }^{4}$ Forschungszentrum Jülich, Institut für Kernphysik (Theorie), D-52425 Jülich, Germany \\ ${ }^{5}$ Institute of Nuclear Physics, Academy of Sciences, Uzbekistan
}

(Received 6 December 2004; published 31 March 2005)

\begin{abstract}
We consider the spin-isospin-independent central part of the residual nucleon-nucleon potential in finite spherical nuclei taking into account the deformation effects of the nucleons within the surrounding nuclear environment. It is shown that inside the nucleus the short-range repulsive contribution of the potential is increased and the intermediate-range attraction is decreased. We identify the growth of the radial component of the spin-isospin-independent short-range part of the in-medium nucleon-nucleon interaction as the responsible agent that prevents the radial collapse of the nucleus.
\end{abstract}

DOI: 10.1103/PhysRevC.71.034007

PACS number(s): 12.39.Dc, 12.39.Fe, 21.30.Fe

\section{INTRODUCTION}

Nearly 20 years ago, in their pioneering work [1], Jackson, Jackson, and Pasquier investigated the skyrmion-skyrmion interaction by considering simple deformations of the chiral field. This idea was further developed in Refs. [2-4] into various versions of Skyrme-like models allowing for more complicated deformations. It was shown that the central potential of the original Skyrme model is reduced by an amount of about $10-20 \%$ if deformation effects of the skyrmion are taken into account. In the $\omega$-stabilized Skyrme model the deformation effect is even more significant, as the central repulsion at $R=1 \mathrm{fm}$ acquires a reduction of about $40 \%$.

Nowadays, in-medium properties of the nucleon are of high relevance. In particular, the properties of a single nucleon embedded in various finite nuclei have been considered in a medium-modified version of the Skyrme model $[5,6]$. It has been shown that bound nucleons acquire an intrinsic quadrupole moment because of the in-medium deformation effects. In summary, the general behavior of the properties of the nucleons are in qualitative agreement with experimental observations (e.g., the swelling of the nucleon in the nuclear medium and the decrease of its mass). Similar results have also been obtained in the infinite-nuclear-matter approach of Ref. [7].

Thus, it is natural to investigate the nucleon-nucleon $(N N)$ interactions in the nuclear medium because of their role in the formation of nuclear matter. There are two known paths to nuclear matter aspects of the Skyrme model. The first one is based on studies of the crystalline ground state of the skyrmion matter [8-16], whereas the second one is related to studies of the Skyrme model on the hypersphere [17-23]. Especially in the first approach, the tensor part of the potential is found to be responsible for the crystalline structure of the ground state. In contrast to this, quantum hadrodynamics studies [24] assert the important role of the spin-isospin independent scalar part of the $N N$ interactions in the formation of the nuclear matter. In any case, one can expect that the surrounding nuclear environment will leave its mark on the in-medium $N N$ potential.

The temperature and density dependence of the nucleonnucleon interactions, the hadron properties and the mesonnucleon coupling constants of the one-boson-exchange potential have been studied in the framework of thermofield dynamics (TFD) in Refs. [25-29]. In particular, it has been shown that the potential well of the $N N$ interaction becomes shallow as the temperature or the density increases [25]. The changes in the meson-nucleon couplings and hadron properties also indicate the influence of the medium on the one-bosonexchange potential $[28,29]$. Note, however, that the Skyrme model and its variants are the only type of models that use hadronic degrees of freedom and that allow for a simultaneous description of both single-baryon and multibaryon properties. In fact, as discussed in Ref. [5], Skyrme-type models even allow for a simple inclusion of nuclear background terms.

The alterations of the tensor part of the $N N$ potential for infinite nuclear matter have already been studied in the framework of the in-medium modified Skyrme model in Ref. [7]. In the present article, we rather concentrate on the spin-isospin-independent part of the $N N$ potential in finite nuclei where we allow the chiral fields of the two involved nucleons to deform in response to the surrounding nuclear environment.

\section{THE MODEL}

Unfortunately, the $N N$ potential of the original Skyrme model does not have a central attraction at intermediate distances. It has been shown, however, that this long-standing problem can be solved, for example, by the incorporation of the iterated two-pion exchange $[30,31]$ into the Skyrme model or by a reformulation [32] of the original Skyrme Lagrangian on the basis of scale invariance and the conformal anomaly 
$[33,34]$. Such a scale-invariant Lagrangian was already applied to describe nucleons in infinite nuclear matter $[35,36]$ in the mean-field approximation (MFA).

\section{A. Lagrangian and baryon number one soliton}

We start here with the model of Refs. [37] that proposes a scale-invariant version of the in-medium Skyrme model given, in the static case, by the following Lagrangian:

$$
\begin{aligned}
\mathcal{L}_{\mathrm{st}}(U, \sigma)= & -\frac{F_{\pi}^{2}}{16}\left[\chi^{2} \alpha_{p}(\vec{x}) \operatorname{Tr}\left(\vec{\nabla}_{\vec{x}} U\right)\left(\vec{\nabla}_{\vec{x}} U^{+}\right)+2\left(\vec{\nabla}_{\vec{x}} \chi\right)^{2}\right] \\
& +\frac{1}{32 e^{2}} \operatorname{Tr}\left[U^{+} \frac{\partial}{\partial x_{i}} U, U^{+} \frac{\partial}{\partial x_{j}} U\right]^{2} \\
& -\frac{C_{g}^{*}}{24}\left[\chi^{4}-1+\frac{4}{\varepsilon}\left(1-\chi^{\varepsilon}\right)\right] \\
& -\frac{F_{\pi}^{2} m_{\pi}^{2}}{16} \chi^{3} \alpha_{s}(\vec{x}) \operatorname{Tr}\left(2-U-U^{+}\right) .
\end{aligned}
$$

Here, $U=\exp \left\{2 i \vec{\tau} \cdot \vec{\pi} / F_{\pi}\right\}$ parametrizes the pseudoscalar isotriplet of pion fields $\vec{\pi}, \vec{\tau}$ are the usual Pauli matrices, $\chi=e^{-2 \sigma / F_{\pi}}$ is given in terms of the scalar-isoscalar dilaton field $\sigma, F_{\pi}$ is the weak pion decay constant, $e$ is the parameter of the stabilizing Skyrme term, $m_{\pi}$ is the pion mass, $\varepsilon=16 / 29$, and $C_{g}^{*}$ is the gluon-condensate parameter. ${ }^{1}$ We associate the dilaton with a quarkonium state. Its mass $m_{\sigma} \approx 600 \mathrm{MeV}$ is compatible with the well-known attraction in the central $N N$ potential [32]. The skyrmion is assumed to be located at a position $\vec{R}_{k}$ from the center of the nucleus, such that the total spatial vector measured relative to the center of the nucleus is given as $\vec{x}=\vec{R}_{k}+\vec{r}$, where $\vec{r}$ is the distance vector relative to the origin of the skyrmion. ${ }^{2}$ We have chosen this model as a representative for a larger class of Skyrme-type models that allow for a qualitative description of the central attraction between two nucleons. It is not our aim to construct a fine-tuned version that produces quantitative fits of the single nucleon properties or the two-nucleon potentials. Rather, by keeping the involved terms to the bare minimum for this case (i.e., the nonlinear sigma-model kinetic term, the stabilizing fourth-order derivative term, the symmetry-breaking pion mass term, and the intermediate-attraction generating dilaton term and couplings) this model should be general enough to predict qualitatively those results which are generic and also hold for more complicated Skyrme-type Lagrangians (e.g., with vector mesons and other hadronic fields).

The dependence on the nuclear density $\rho(\vec{x})$ is included in the in-medium coefficients $\alpha_{s}(\vec{x})$ and $\alpha_{p}(\vec{x})$ as follows:

$$
\begin{aligned}
& \alpha_{p}(\vec{x})=1-\frac{4 \pi c_{0} \rho(\vec{x}) / \eta}{1+g_{0}^{\prime} 4 \pi c_{0} \rho(\vec{x}) / \eta}, \\
& \alpha_{s}(\vec{x})=1-4 \pi \eta b_{0} \rho(\vec{x}) / m_{\pi}^{2} .
\end{aligned}
$$

\footnotetext{
${ }^{1}$ The asterisk indicates that the gluon condensate should be considered as renormalized in the nuclear medium. Note that in general $\varepsilon=8 N_{f} /\left(11 N_{c}-2 N_{f}\right)$. Furthermore, we assume $F_{\pi}=F_{\sigma}$.

${ }^{2}$ This corresponds to $U=U\left(\vec{x}-\vec{R}_{k}\right)=U(\vec{r})$ and $\chi=\chi(\vec{x}-$ $\left.\vec{R}_{k}\right)=\chi(\vec{r})$ in Eq. (1). See Ref. [5] for further details on the geometry of a skyrmion (without dilaton) inside a finite nucleus.
}

Here $\eta=1+m_{\pi} / m_{N} \sim 1.14$ is a kinematical factor and $m_{N}=938 \mathrm{MeV}$ is the mass of the nucleon. Moreover, $b_{0}=$ $-0.024 m_{\pi}^{-1}$ and $c_{0}=0.21 m_{\pi}^{-3}$ are empirical parameters that can be taken from the analyses of pionic atoms and low-energy pion-nucleus scattering data, and $g_{0}^{\prime}=1 / 3$ is the Lorentz-Lorenz factor that takes into account the short-range correlations [38].

Numerical calculations show that the ground state $B=1$ solution of the Euler-Lagrange equations of (1) in free space ( $\left.\rho=0, \alpha_{s}=\alpha_{p}=1\right)$ is spherically symmetric. However, when the soliton is embedded into a finite nucleus, it may alter its shape, because the spherical symmetric configuration may not correspond to a minimum in energy or mass any longer. In this way it is possible to study the modification of the shape of the in-medium skyrmion, namely by a minimization procedure of its mass functional.

In deriving the mass functional of the single skyrmion we follow the scheme presented in Ref. [5] by considering an axially symmetric configuration for a single skyrmion located at some distance from the center of a finite spherical nucleus. This implies that the chiral profile function, which parametrizes the modulus of the pion, is axially symmetric, that is, $F(\vec{r})=F(r, \theta)$, whereas the pion direction is governed by the polar-angle profile function $\Theta(\theta)$ :

$$
\begin{aligned}
\vec{\pi}(\vec{r}) & =\frac{F_{\pi}}{2} F(r, \theta) \vec{N}[\Theta(\theta), \varphi], \\
\vec{N} & =\{\sin \Theta(\theta) \cos \varphi, \sin \Theta(\theta) \sin \varphi, \cos \Theta(\theta)\} .
\end{aligned}
$$

We further assume that the dilaton field also has an axially symmetric configuration, that is, $\sigma(\vec{r})=\sigma(r, \theta)$ and therefore $\chi(\vec{r})=\chi(r, \theta)$. For example, the mass functional for a single skyrmion $(B=1)$ located at a distance $R_{k}=\left|\vec{R}_{k}\right|$ from the center of the nucleus, and with a spatial vector $\vec{r}_{k}$ measured relative to the origin of the skyrmion, ${ }^{3}$ is given as follows:

$$
\begin{aligned}
M\left(R_{k}\right)= & 2 \pi \int_{0}^{\infty} d r_{k} r_{k}^{2} \int_{0}^{\pi} d \theta_{k} \sin \theta_{k} \\
& \times\left\{\frac{F_{\pi}^{2}}{8} \phi\left(F, \Theta ; r_{k}, \theta_{k}\right) \chi^{2} \alpha_{p}\left[x\left(r_{k}, \theta_{k} ; R_{k}\right)\right]\right. \\
& +\frac{1}{2 e^{2}} \varphi\left(F, \Theta ; r_{k}, \theta_{k}\right) \\
& +\frac{F_{\pi}^{2} m_{\pi}^{2}}{4}(1-\cos F) \chi^{3} \alpha_{s}\left[x\left(r_{k}, \theta_{k} ; R_{k}\right)\right] \\
& \left.+\frac{C_{g}^{*}}{24} \psi(\chi)+\frac{F_{\pi}^{2}}{8}\left(\frac{\chi_{\theta_{k}}^{2}}{r_{k}^{2}}+\chi_{r_{k}}^{2}\right)\right\} \\
\phi\left(F, \Theta ; r_{k}, \theta_{k}\right)= & \frac{F_{\theta_{k}}^{2}}{r_{k}^{2}}+F_{r_{k}}^{2}+\frac{\sin ^{2} F}{r_{k}^{2}}\left(\frac{\sin ^{2} \Theta}{\sin ^{2} \theta_{k}}+\Theta_{\theta_{k}}^{2}\right),
\end{aligned}
$$

\footnotetext{
${ }^{3}$ Note that here $\vec{r}=\vec{r}_{k}$, where $k$ labels the skyrmion. Thus $\vec{x}=\vec{r}_{k}+$ $\vec{R}_{k}$ and $|\vec{x}|=\sqrt{R_{k}^{2}+r_{k}^{2}+2 R_{k} r_{k} \cos \theta_{k}}=x\left(r_{k}, \theta_{k} ; R_{k}\right)$, where $r_{k}$ and $\theta_{k}$ are the modulus and polar angle of $\vec{r}_{k}$.
} 


$$
\begin{aligned}
\varphi\left(F, \Theta ; r_{k}, \theta_{k}\right)= & \frac{\sin ^{2} F}{r_{k}^{2}}\left[\left(\frac{\sin ^{2} \Theta}{\sin ^{2} \theta_{k}}+\Theta_{\theta_{k}}^{2}\right) F_{r_{k}}^{2}\right. \\
& \left.+\frac{\sin ^{2} \Theta}{\sin ^{2} \theta_{k}}\left(\frac{F_{\theta_{k}}^{2}}{r_{k}^{2}}+\frac{\sin ^{2} F}{r_{k}^{2}} \Theta_{\theta_{k}}^{2}\right)\right], \\
\psi(\chi)= & {\left[\chi^{4}-1+\frac{4}{\varepsilon}\left(1-\chi^{\varepsilon}\right)\right] . }
\end{aligned}
$$

Here the subscripts of the functions $F_{r_{k}}, F_{\theta_{k}}, \Theta_{\theta_{k}}, \chi_{r_{k}}$, and $\chi_{\theta_{k}}$ denote the corresponding partial derivatives [e.g., $\chi_{\theta_{k}} \equiv$ $\partial \chi\left(r_{k}, \theta_{k}\right) / \partial \theta_{k}$ of the function $\left.\chi \equiv \chi\left(r_{k}, \theta_{k}\right)\right]$.

In Ref. [5] the minimization of the mass functional equation [Eq. (4)] was performed without dilatons, that is, $\sigma\left(\vec{r}_{k}\right)=0$, and the pertinent in-medium skyrmion properties were discussed. In particular, it was shown that the medium effects cause a deformation of the nucleon inside finite nuclei. We follow Ref. [5] in the minimization procedure for the functional equation [Eq. (4)]. But in addition to the following parametrizations of the chiral and polar-angle profile functions [5]:

$$
\begin{aligned}
& F=2 \arctan \left\{\left(\frac{r_{S}^{2}}{r_{k}^{2}}\right)\left[1+\gamma_{1} \cos \theta_{k}+\gamma_{2} \cos ^{2} \theta_{k}+\cdots\right]\right\}, \\
& \Theta=\theta_{k}+\delta_{1} \sin 2 \theta_{k}+\delta_{2} \sin 4 \theta_{k}+\delta_{3} \sin 6 \theta_{k}+\cdots,
\end{aligned}
$$

we also introduce the following parametrization of the dilaton field:

$\chi=1-\chi_{d} \exp \left\{-\left(\frac{r_{k}^{2}}{r_{d}^{2}}\right)\left(1+\eta_{1} \cos \theta_{k}+\eta_{2} \cos ^{2} \theta_{k}+\cdots\right)\right\}$.

Here $r_{S}, \gamma_{i}, \delta_{i}, \chi_{d}, r_{d}$, and $\eta_{i}$ are variational parameters. As a result of the minimization procedure one gets a set of variational parameters corresponding to specified values of the distance $R_{k}$ and the other input parameters defined in Secs. II C and III.

\section{B. Two nucleons in a baryon-rich environment and the residual $N N$ interaction}

We now turn to the system of two interacting in-medium nucleons, for example, to the modification of $N N$ potential $V_{N N}$ inside nuclei. For simplicity, we consider only the residual in-medium $N N$ potential in the radial direction of the nucleus (i.e., the axial symmetric case) such that the two nucleons are subject to different densities. This should be contrasted with the case where the in-medium $N N$ interaction is considered in the azimuthal direction, namely where both nucleons are at the same distance from the center of the nucleus and therefore subject to the same density. This is not a serious limitation because the latter interaction ought to be subdominant to the interaction in the radial direction, as the medium modifications resulting from the changes in the profile functions are known to be small, whereas the changes resulting from the density dependence of the mass functionals are more pronounced [5]. The geometry of this axially symmetric case is presented in Fig. 1.

Unfortunately, in any version of the Skyrme Lagrangian, it is very difficult to derive the binding energies as well as the form factors of even light nuclei directly from the nucleon-nucleon potential $V_{N N}$. For simplicity, in setting up our formalism, we assume the following:

- Each nucleon is bound inside the finite nucleus by a phenomenological averaged potential normally used in shell models in MFA.

- The nucleon distribution (i.e., the nuclear density) is given by a phenomenological formula.

It is believed that the sum of all two-body-as well as possible three-body-interactions can be reexpressed as an effective averaged potential, centered at the origin of the nucleus. Because of the MFA ideology, every nucleon behaves as if it "feels" only this effective potential (spherical-well potential, oscillator, etc.). Yet residual interactions between the nucleons may still exist. These interactions ought to be important for the description of deformations and stabilities of the nuclei.

In the following we will study the residual part of the $N N$ interactions (in radial direction) embedded in the surrounding nuclear environment. We assume that the primary two-nucleon potential in free space is given by the Skyrme model, including dilatons, as described by the Lagrangian [Eq. (1)] with density $\rho=0$ such that $\alpha_{p}=\alpha_{s}=1$. We then "plug" a pair of nucleons into a finite nucleus or, more exactly, we consider them as part of the nucleus. The point is that each of the nucleons can be deformed for the following two reasons, which affect the in-medium potential as well: (1) the presence of the other nucleon or, in other words, the presence of the $N N$ interaction and (2) the presence of the remaining $A-2$ nucleons or, in other words, the presence of medium effects in the nucleus.

The first case was investigated in Refs. [4,39], where the modifications of the nucleons and $V_{N N}$ properties in free space were recorded under possible deformations of the nucleons during their mutual approach. For that purpose the authors introduced a couple of deformation parameters and obtained a modified central potential $V_{N N}^{c}$ by minimizing it at each internucleon distance $R$. The second case, namely the effects of the nuclear medium, is the subject of this article. In principal, we could have followed the procedure of Refs. [4,39], which would be

- to introduce deformation parameters in Lagrangian [Eq. (1)] using an axially symmetric ansatz,

- to construct $V_{N N}^{c}$ from the Lagrangian [Eq. (1)] in the product approximation, and

- to determine the deformation parameters by minimizing $V_{N N}^{c}$ at each $R$.

However, such a procedure would be unnecessarily complicated, especially for the case of the Lagrangian [Eq. (1)] with dilatons. Moreover, it that case one would have to overcome complications resulting from double counting the deformation effects caused by the $N N$ interactions and by medium modifications, respectively. Instead, for simplicity and also for isolating the medium modifications from the vacuum $N N$ interaction effects, we minimize the mass functional equation [Eq. (4)] for each of the two in-medium skyrmions with respect to the corresponding deformation parameters, 
and then we insert these deformed skyrmion profiles into the product ansatz to generate the $N N$ central potential.

In this way, by inserting two skyrmions in the nuclear background of the $A-2$ remaining nucleons, one can define a static skyrmion-skyrmion residual potential as follows:

$$
\begin{aligned}
V_{S S} & (\underbrace{\frac{1}{2 \mid}\left|\vec{R}_{1}+\vec{R}_{2}\right|}_{\equiv D}, \underbrace{\left|\vec{R}_{2}-\vec{R}_{1}\right|}_{\equiv R}) \\
= & M\left[U(\underbrace{\vec{x}-\vec{R}_{1}}_{\equiv \vec{r}_{1}}) U(\underbrace{\vec{x}-\vec{R}_{2}}_{\equiv \vec{r}_{2}}), \chi\left(\vec{r}_{1}\right) \chi\left(\vec{r}_{2}\right) ; \rho_{A-2}(|\vec{x}|)\right] \\
& -2 M_{B=1}^{\text {free }}-\delta V_{B=2}^{M F}(D, R) .
\end{aligned}
$$

Here $M\left[\prod_{i=1}^{2} U\left(\vec{x}-\vec{R}_{i}\right), \prod_{i=1}^{2} \chi\left(\vec{x}-\vec{R}_{i}\right) ; \rho_{A-2}(|\vec{x}|)\right]$ is the static mass functional of a skyrmion-product ansatz of baryon number $B=2$ with the skyrmions centered (axialsymmetrically) at $\vec{R}_{i} \equiv \overrightarrow{\mathcal{O O}}_{i}$ (with $\mathcal{O}$ and $\mathcal{O}_{i}$ as defined in Fig. 1) in the renormalized background density $\rho_{A-2}(|\vec{x}|)$. Thus the potential [Eq. (7)] is defined in analogy with the well-known product-ansatz prescription of the dilaton-free case

$$
U_{B=2}(\vec{x}-\vec{D}, \vec{R})=U\left(\vec{x}-\vec{R}_{1}\right) U\left(\vec{x}-\vec{R}_{2}\right),
$$

where $\vec{D} \equiv\left(\vec{R}_{1}+\vec{R}_{2}\right) / 2$ and $\vec{R} \equiv \vec{R}_{2}-\vec{R}_{1}$. The remaining quantity $\delta V_{B=2}^{M F}$ denotes the contribution of the $B=2$ system to the mean-field potential. It is defined as follows:

$$
\delta V_{B=2}^{M F}=\sum_{i=1}^{2}\left(M\left[U\left(\vec{r}_{i}\right), \chi\left(\vec{r}_{i}\right) ; \rho_{A-2}(|\vec{x}|)\right]-M_{B=1}^{\text {free }}\right) .
$$

Note that the corresponding quantity $\delta M_{S}=-\delta V_{B=1}^{M F}$ can be interpreted as the part of the energy of a skyrmion that is transformed to the mean-field energy of pions inside the nucleus and to the interaction energy of the skyrmion with the background field. It is this background pionic field that generates the attractive mean field potential binding nucleons to the nucleus. Clearly the potential [Eq. (7)] becomes the free $N N$ potential when the background density of the remaining nucleons goes to zero. ${ }^{4}$

Finally, inserting Eq. (9) into Eq. (7), one gets the following form of the skyrmion-skyrmion residual potential:

$$
\begin{aligned}
V_{S S}(D, R)= & M\left[U\left(\vec{r}_{1}\right) U\left(\vec{r}_{2}\right), \chi\left(\vec{r}_{1}\right) \chi\left(\vec{r}_{2}\right) ; \rho_{A-2}(|\vec{x}|)\right] \\
& -M\left[U\left(\vec{r}_{1}\right), \chi\left(\vec{r}_{1}\right) ; \rho_{A-2}(|\vec{x}|)\right] \\
& -M\left[U\left(\vec{r}_{2}\right), \chi\left(\vec{r}_{2}\right) ; \rho_{A-2}(|\vec{x}|)\right] .
\end{aligned}
$$

From the comparison of Eqs. (7), (9), and (10) it should become clear that the potential expressed in Eq. (10) is simply the difference between the separation energy of a pair of skyrmions $(B=2)$ inside the nucleus and the sum of the separation energies of each individual skyrmion $(B=1)$ in the same nucleus. This is well known in nuclear physics as a major contribution to the residual $N N$ potential in the nuclear medium. Our aim is the evaluation of the spin-isospin independent part of this potential.

\footnotetext{
${ }^{4}$ If the background field vanishes, $\rho_{A-2} \rightarrow 0$, the mean-field contribution also vanishes, $\delta V_{B=2}^{M F} \rightarrow 0$.
}

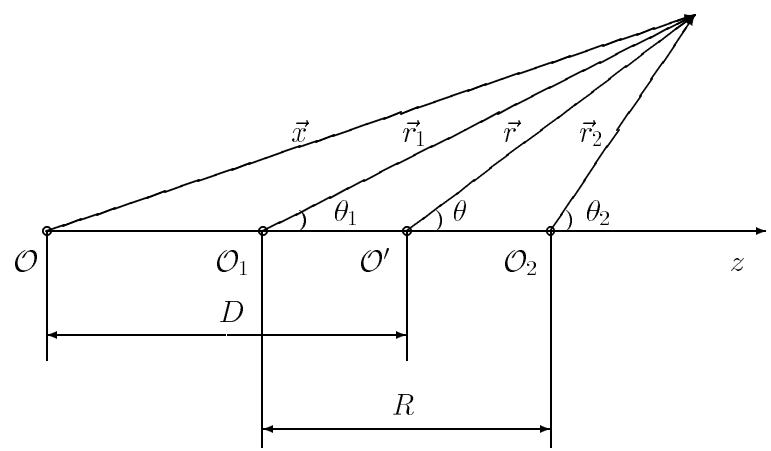

FIG. 1. The axially symmetric two-skyrmion system in a finite spherical nucleus. Here $\mathcal{O}$ is the center of the nucleus, $\mathcal{O}^{\prime}$ is the geometrical center of the two-skyrmion system, and $\mathcal{O}_{1}$ and $\mathcal{O}_{2}$ are the centers of the first and second skyrmions, respectively. $D$ is the distance between the center of the nucleus and the one of the twoskyrmion system, and $R$ is the distance between skyrmions.

As mentioned already, we only consider the geometry when the center of the (spherical) nucleus and centers of the two skyrmions fall onto the same axis, which is denoted by $z$ as illustrated in Fig. 1. Then the $V_{N N}$ potential depends on only two variables: (1) the distance between the centers of the two skyrmions, $R$, and (2) the distance between the geometrical center of the two-skyrmion system and the center of the nucleus, $D$. The spatial vector $\vec{r}$ is measured relative to the two-skyrmion center such that here $\vec{x}=\vec{D}+\vec{r}$.

To isolate the spin-isospin independent part of the $N N$ potential one can use the standard projection procedure [40], which corresponds to the following form in free space:

$$
\begin{aligned}
V_{N N}(R, C)= & V_{N N}^{c}+\frac{1}{2} \operatorname{Tr}\left(C \sigma_{i} C^{+} \sigma_{j}\right)\left[\left(3 \hat{R}_{i} \hat{R}_{j}-\delta_{i j}\right)\right. \\
& \left.\times V_{N N}^{T}(R)+\delta_{i j} V_{N N}^{\sigma \tau}(R)\right],
\end{aligned}
$$

where $C=u_{0}+i \vec{\tau} \cdot \vec{u}$ (with $\vec{u}^{2}+u_{0}^{2}=1$ ) is the relative orientation matrix of the nucleons in internal space, $\sigma_{i}, \sigma_{j}$ are the Pauli matrixes, and $\hat{R}$ is the unit vector along the line joining the centers of the two nucleons. Here $V_{N N}^{c}, V_{N N}^{T}$, and $V_{N N}^{\sigma \tau}$ are the central, tensor, and spin-spin parts of the $N N$ potential, respectively. Note that the central part of the nucleon-nucleon interaction coincides with the one of the skyrmion-skyrmion interaction.

Applying the above-described projection procedure and using the skyrmion-skyrmion potential [Eq. (10)], we therefore predict the following expression for the spin-isospinindependent part of the residual $N N$ potential (in radial direction) inside a finite spherical nucleus:

$V_{N N}^{c, A}(D, R)=2 \pi \int_{0}^{\infty} d r r^{2} \int_{0}^{\pi} d \theta \sin \theta\left\{V_{2}^{c}+V_{4}^{c}+V_{\chi S B}^{c}+V_{\sigma}^{c}\right\}$

with

$$
\begin{aligned}
V_{2}^{c}= & \frac{F_{\pi}^{2}}{8} \alpha_{p, A-2}[x(r, \theta ; D)]\left[\chi_{1}^{2} \chi_{2}^{2}\left(\phi_{1}+\phi_{2}\right)\right. \\
& \left.-\left(\chi_{1}^{2} \phi_{1}+\chi_{2}^{2} \phi_{2}\right)\right], \\
V_{4}^{c}= & \frac{1}{3 e^{2}}\left(\phi_{1} \phi_{2}-\Phi\right),
\end{aligned}
$$




$$
\begin{aligned}
& V_{\chi S B}^{c}=\frac{m_{\pi}^{2} F_{\pi}^{2}}{4} \alpha_{s, A-2}[x(r, \theta ; D)]\left[\chi_{1}^{3} \chi_{2}^{3}\left(1-\cos F_{1} \cos F_{2}\right)\right. \\
& \left.-\chi_{1}^{3}\left(1-\cos F_{1}\right)-\chi_{2}^{3}\left(1-\cos F_{2}\right)\right] \text {, } \\
& V_{\sigma}^{c}=\frac{C_{g, A-2}^{*}}{24}\left[\psi\left(\chi_{1} \cdot \chi_{2}\right)-\psi\left(\chi_{1}\right)-\psi\left(\chi_{2}\right)\right] \\
& +\frac{F_{\pi}^{2}}{8}\left(\chi_{1}^{2}-1\right)\left(\frac{\chi_{2, \theta_{2}}^{2}}{r_{2}^{2}}+\chi_{2, r_{2}}^{2}\right) \\
& +\frac{F_{\pi}^{2}}{8}\left(\chi_{2}^{2}-1\right)\left(\frac{\chi_{1, \theta_{1}}^{2}}{r_{1}^{2}}+\chi_{1, r_{1}}^{2}\right) \\
& +\frac{F_{\pi}^{2}}{4} \chi_{1} \chi_{2}\left[\sin \left(\theta_{1}-\theta_{2}\right)\left(\chi_{1, r_{1}} \frac{\chi_{2, \theta_{2}}}{r_{2}}-\frac{\chi_{1, \theta_{1}}}{r_{1}} \chi_{2, r_{2}}\right)\right. \\
& \left.+\cos \left(\theta_{1}-\theta_{2}\right)\left(\chi_{1, r_{1}} \chi_{2, r_{2}}+\frac{\chi_{1, \theta_{1}} \chi_{2, \theta_{2}}}{r_{1} r_{2}}\right)\right] \text {, } \\
& \Phi=\left[\sin \left(\theta_{1}-\theta_{2}\right)\left(F_{1, r_{1}} \frac{F_{2, \theta_{2}}}{r_{2}}-\frac{F_{1, \theta_{1}}}{r_{1}} F_{2, r_{2}}\right)\right. \\
& \left.+\cos \left(\theta_{1}-\theta_{2}\right)\left(F_{1, r_{1}} F_{2, r_{2}}+\frac{F_{1, \theta_{1}} F_{2, \theta_{2}}}{r_{1} r_{2}}\right)\right]^{2} \\
& +\frac{\sin ^{2} F_{1}}{r_{1}^{2}}\left[\cos \left(\theta_{1}-\theta_{2}\right) \frac{F_{2, \theta_{2}}}{r_{2}}\right. \\
& \left.-\sin \left(\theta_{1}-\theta_{2}\right) F_{2, r_{2}}\right]^{2} \Theta_{1, \theta_{1}}^{2} \\
& +\frac{\sin ^{2} F_{2}}{r_{2}^{2}}\left(\cos \left(\theta_{1}-\theta_{2}\right) \frac{F_{1, \theta_{1}}}{r_{1}}\right. \\
& \left.+\sin \left(\theta_{1}-\theta_{2}\right) F_{1, r_{1}}\right)^{2} \Theta_{2, \theta_{2}}^{2} \\
& +\frac{\sin ^{2} F_{1}}{r_{1}^{2}} \frac{\sin ^{2} F_{2}}{r_{2}^{2}}\left[\frac{\sin ^{2} \Theta_{1}}{\sin ^{2} \theta_{1}} \frac{\sin ^{2} \Theta_{2}}{\sin ^{2} \theta_{2}}\right. \\
& \left.+\cos ^{2}\left(\theta_{1}-\theta_{2}\right) \Theta_{1, \theta_{1}}^{2} \Theta_{2, \theta_{2}}^{2}\right] \text {. }
\end{aligned}
$$

Here $F_{i} \equiv F_{i}\left(r_{i}, \theta_{i}\right), \chi_{i} \equiv \chi_{i}\left(r_{i}, \theta_{i}\right)$, and $\Theta_{i} \equiv \Theta_{i}\left(\theta_{i}\right)$ with $i=1,2$ are the chiral, dilaton, and polar-angle profile functions of skyrmion 1 or 2 , respectively. The variables $r_{i}=r_{i}(r, \theta ; R)=\sqrt{\left(R^{2} / 4\right)+r^{2} \pm R r \cos \theta}, \theta_{i}=\theta_{i}(r$, $\theta ; R)=\arcsin \left[\sin \theta r / r_{i}(r, \theta ; R)\right], r, \theta$ and $x=x(r, \theta ; D)=$ $\sqrt{D^{2}+r^{2}+2 D r \cos \theta}$ are illustrated in Fig. 1. Moreover, we abbreviate the partial derivative of the above-specified functions $f_{i} \in\left\{F_{i}, \chi_{i}, \Theta_{i}\right\}$ as $f_{i, y} \equiv \partial f_{i} / \partial y$. The functions $\phi_{i}$ are defined as $\phi_{i} \equiv \phi\left(F_{i}, \Theta_{i} ; r_{i}, \theta_{i}\right)$ with $\phi($ and $\psi)$ as in Eq. (4).

\section{Density dependence and input parameters}

We shall use a phenomenological parametrization of the density of a spherical nucleus as presented in Ref. [41] and therefore write the renormalized density of the $A-B$ nuclear background environment as follows:

$$
\begin{aligned}
\rho_{A-B}(x)= & \left(\frac{A-B}{A}\right) \frac{2}{\pi^{3 / 2} r_{0}^{3}}\left[1+\frac{A-2}{3}\left(\frac{x^{2}}{r_{0}^{2}}\right)\right] \\
& \times \exp \left\{-\frac{x^{2}}{r_{0}^{2}}\right\} \quad \text { if } A<20,
\end{aligned}
$$

$$
\begin{aligned}
\rho_{A-B}(x)= & \left(\frac{A-B}{A}\right) \frac{\rho_{0}}{1+\exp \left\{\left(x-R^{\prime}\right) / a\right\}} \\
& \text { if } A \geqslant 40,
\end{aligned}
$$

where $r_{0}=1.635 \mathrm{fm}$ for ${ }^{12} \mathrm{C}$. Furthermore, $a=0.58 \mathrm{fm}$, $R^{\prime}=1.2 A^{1 / 3} \mathrm{fm}$, and $\rho_{0}=0.5 m_{\pi}^{3}$ is the normal nuclear matter density.

The input parameters of the skyrmion sector are chosen as $F_{\pi}=186 \mathrm{MeV}$ and $e=2 \pi$. Although this standard set of input parameters gives an overestimated value for the mass of nucleon even in free space, we shall not optimize it, because we are interested only in the influence of the medium on the $N N$ potential. Furthermore, the results presented in Sec. III do not qualitatively change if these parameters are varied to fit, for example, the nucleon mass or the axial-vector coupling constant. The sole input parameter in the dilaton sector is the gluon condensate parameter $C_{g}^{*}$. Its in-medium renormalization has not been clarified in the literature. In the present model this quantity can be expressed in terms of the in-medium sigma-meson mass $m_{\sigma}^{*}$ as $[34,37]$

$$
C_{g, A-B}^{*}=\frac{3 F_{\pi}^{2} m_{\sigma, A-B}^{* 2}}{2(4-\varepsilon)} .
$$

Unfortunately, no information exists about the renormalization of $m_{\sigma}$ within the present approach. However, other models $[36,42,43]$ predict a linear density dependence for $m_{\sigma}^{*}$. In view of this, we shall use the following parametrization:

$$
m_{\sigma, A-B}^{*}(x)=\left[1-0.12 \frac{\rho_{A-B}(x)}{\rho_{0}}\right] m_{\sigma},
$$

which was obtained in Ref. [43] within the quark-meson coupling model. ${ }^{5}$ The free-space mass of the sigma meson is taken as $m_{\sigma}=550 \mathrm{MeV}$, which corresponds to a free-space value of $C_{g}=(260 \mathrm{MeV})^{4}$.

\section{RESULTS AND DISCUSSION}

The results of the minimization procedure for a single skyrmion $(B=1)$ in the nucleus with an $A-1$ background density is presented in Table I. We have not listed all variational parameters, as some of them are rather small, namely $\gamma_{i}, \delta_{i}, \eta_{i}$ for $i \geqslant 3$. These parameters, which describe higher multipole deviations of the skyrmion shape from the spherical form, can be neglected without loss of accuracy. A discussion about the parameters of the chiral sector can be found in Ref. [5], whereas the ones of the dilaton sector are new. Both sets are consistent with the fact that the nucleon inside the nucleus acquires an intrinsic quadrupole moment unless the nucleon is located directly at the center. Furthermore, note that the increase of the radial parameter $r_{S}$ of the chiral sector and of the radial parameter $r_{d}$ of the dilaton sector with increasing density is in qualitative agreement with the overall picture of the swelling of the nucleon inside the nucleus. The behavior of the depth of the dilaton field parametrized by $\chi_{d}$ is more

\footnotetext{
${ }^{5}$ Because in finite nuclei the density is coordinate dependent, $m_{\sigma}^{*}$ will also acquire a coordinate dependence.
} 
TABLE I. Deformation parameters at various separations $R_{k}$ of the center of a single skyrmion from the center of the nucleus. The parameters $r_{S}, \gamma_{i}, \delta_{i}$ are for the chiral sector, whereas $\chi_{d}, r_{d}, \eta_{i}$ are for the dilaton sector. They are obtained by a direct minimization of the mass functional in Eq. (4). Note that all coefficients $\gamma_{i}, \delta_{i}, \eta_{i}$ for $i \geqslant 3$ are negligible and are not presented here. Nonzero free-space values of some parameters are $r_{S}=0.398 \mathrm{fm}, \chi_{d}=0.877$, and $r_{d}=0.740 \mathrm{fm}$.

\begin{tabular}{|c|c|c|c|c|c|}
\hline \multicolumn{6}{|c|}{${ }^{12} \mathrm{C}$} \\
\hline$R_{k}(\mathrm{fm})$ & 0 & \pm 0.649 & \pm 1.298 & \pm 1.947 & \pm 2.595 \\
\hline$r_{S}(\mathrm{fm})$ & 0.512 & 0.509 & 0.495 & 0.467 & 0.435 \\
\hline$\gamma_{1}$ & 0 & \pm 0.020 & $\mp 0.034$ & $\mp 0.105$ & $\mp 0.107$ \\
\hline$\gamma_{2}$ & 0 & -0.028 & -0.047 & 0.001 & 0.037 \\
\hline $10 \delta_{1}$ & 0 & -0.056 & -0.097 & -0.018 & 0.047 \\
\hline $10 \delta_{2}$ & 0 & 0 & -0.002 & -0.003 & -0.001 \\
\hline$\chi_{d}$ & 0.634 & 0.629 & 0.592 & 0.655 & 0.766 \\
\hline$r_{d}(\mathrm{fm})$ & 0.843 & 0.841 & 0.835 & 0.808 & 0.776 \\
\hline$\eta_{1}$ & 0 & \pm 0.055 & \pm 0.008 & $\mp 0.060$ & $\mp 0.054$ \\
\hline$\eta_{2}$ & 0 & -0.015 & -0.032 & 0 & 0.017 \\
\hline \multicolumn{6}{|c|}{${ }^{40} \mathrm{Ca}$} \\
\hline$R_{k}(\mathrm{fm})$ & 0 & \pm 1.705 & \pm 3.409 & \pm 5.114 & \pm 6.819 \\
\hline$r_{S}(\mathrm{fm})$ & 0.597 & 0.576 & 0.477 & 0.407 & 0.398 \\
\hline$\gamma_{1}$ & 0 & $\mp 0.053$ & $\mp 0.145$ & $\mp 0.035$ & $\mp 0.002$ \\
\hline$\gamma_{2}$ & 0 & -0.011 & 0.030 & 0.017 & 0.001 \\
\hline $10 \delta_{1}$ & 0 & -0.026 & 0.021 & 0.027 & 0.002 \\
\hline $10 \delta_{2}$ & 0 & 0 & -0.001 & 0 & 0 \\
\hline$\chi_{d}$ & 0.397 & 0.423 & 0.650 & 0.854 & 0.876 \\
\hline$r_{d}(\mathrm{fm})$ & 0.983 & 0.951 & 0.818 & 0.749 & 0.741 \\
\hline$\eta_{1}$ & 0 & $\mp 0.020$ & $\mp 0.076$ & $\mp 0.010$ & $\mp 0.001$ \\
\hline$\eta_{2}$ & 0 & -0.005 & 0.014 & 0.004 & 0 \\
\hline
\end{tabular}

involved, but at least for the case of the heavier nucleus, the dilaton region of the in-medium nucleon becomes shallower with increasing density. At first sight this is counterintuitive. However, note that because of the swelling of the nucleon, the total region where the "effective pion decay parameter" $\chi F_{\pi}$ is reduced relatively to its vacuum value $F_{\pi}$ has increased nevertheless.

The results of the calculations for the residual $N N$ interactions are summarized in Figs. 2 and 3. The spin-isospinindependent central part $V_{N N}^{c}$ of the residual $N N$ potential in ${ }^{12} \mathrm{C}$ is shown in Fig. 2. Our model has not been fine tuned to reproduce the spin-isospin-independent $N N$ potential in free space quantitatively. But the qualitative changes are evident: One can see that short-range repulsive contribution of the potential is increased and the intermediate attraction is decreased in the nuclear medium. The same behavior of $V_{N N}^{c}$ is observed for skyrmions embedded in ${ }^{40} \mathrm{Ca}$, as shown in Fig. 3.

It is known that the residual $N N$ potential becomes repulsive when neighboring nucleons overlap. It is furthermore known that the sizes of the nucleons increase in the medium; see also the parameters $r_{S}$ and $r_{d}$ of Table I. Therefore the overlap regions increase with density. This leads to a buildup of the repulsive part of the potential at high densities (i.e., when the two-nucleon system is near the center of the nucleus).

From previous studies [5] we know that the nucleon mass is the smallest near the center of nucleus and increases when the nucleon is moved to the surface. Because of this phenomenon, nucleons should actually collapse to the center of the nucleus. In other words, because of the attractive mean-field potential, nucleons should move to the center. Conversely, the nucleon

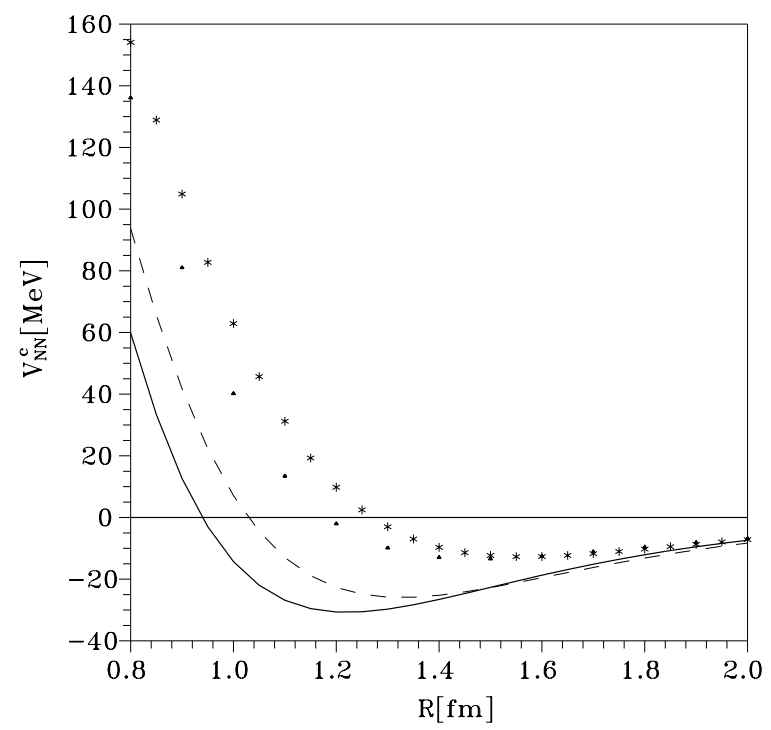

FIG. 2. Spin-isospin-independent central part (in the radial direction) of the residual $N N$ potential in ${ }^{12} \mathrm{C}$. The solid line corresponds to the potential in the free case, the dashed line corresponds to $D=$ $3 \mathrm{fm}$, the dotted line corresponds to $D=2 \mathrm{fm}$, and the stars correspond to $D=0 \mathrm{fm}$. 


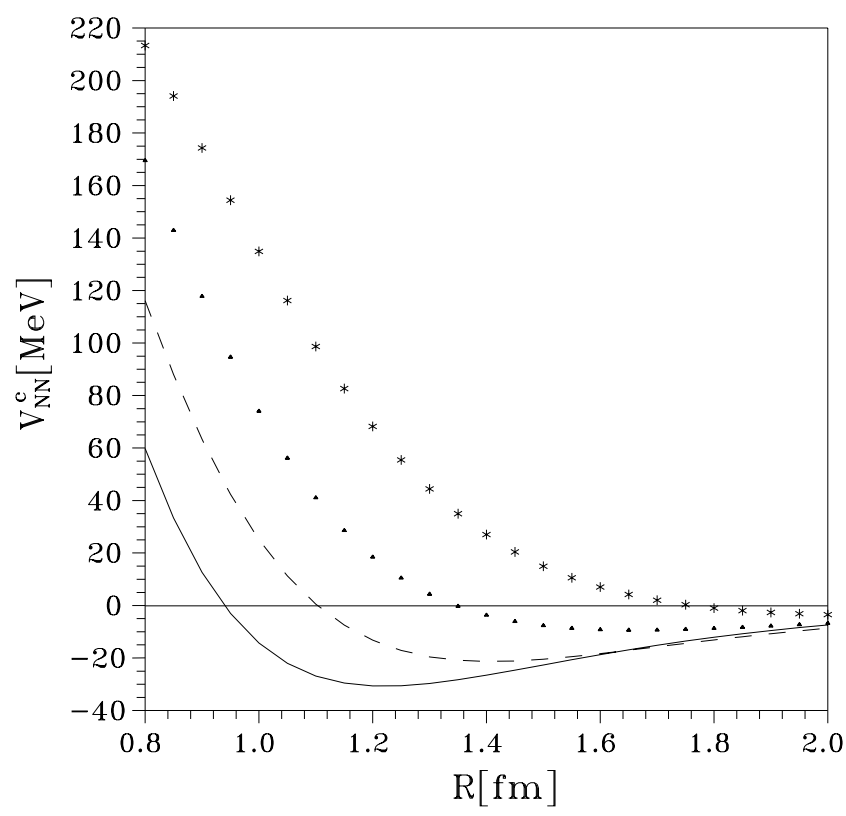

FIG. 3. Spin-isospin-independent central part (in radial direction) of the residual $N N$ potential in ${ }^{40} \mathrm{Ca}$. The solid line corresponds to the potential in the free case, the dashed line corresponds to $D=$ $4 \mathrm{fm}$, the dotted line corresponds to $D=3 \mathrm{fm}$, and the stars correspond to $D=0 \mathrm{fm}$.

concentration near the center of the nucleus does, as shown in Figs. 2 and 3, increase the repulsive residual potentials between nucleons such that nucleons are expelled from the center. Consequently, an equilibrium state arises and therefore saturation of the nuclear matter density results. Thus the nucleons stop their radial motion toward the center of the nucleus, but their angular motion in a shell of a given radius continues. An inclusion of the Pauli mechanism in this picture could finally provide for the shell description of finite nuclei.

Su et al. obtained similar results to ours in the framework of a chiral $\sigma-\omega$ model under the imaginary-time Green's function method [44]. They found that the potential well of the nucleon-nucleon interaction becomes shallow as the temperature increases. The same behavior of the $N N$ potential was seen in quantum hadrodynamics studies [45] and in the framework of TFD [25]. The latter established, in addition, the alternative roles of density and temperature effects.

\section{SUMMARY AND OUTLOOK}

We have considered the the central part of the residual $N N$ potential inside finite nuclei (in the radial direction) in the framework of a dilaton-extended Skyrme model.
We have shown that in the interior region of the heavy nucleus the residual $N N$ potential is strongly repulsive. It therefore compensates the mean-field attractive potential and the reduction of the in-medium nucleon mass in a such way that the nuclear density has to saturate. These results are consistent with other studies applying different methods [25,44,45]. However, one should remember that the Skyrme-type models are the only models that are formulated in terms of hadronic degrees of freedom (without quarks and gluons) and that can simultaneously describe both single-baryon as well as multibaryon properties. In the framework of such a class of models our results are indeed new.

It would be interesting to investigate the role of the residual interactions in the formation of nuclear matter itself (i.e., to study their role in the formation of the mean-field potential). This requires the additional study of the noncentral parts of the in-medium $N N$ potential. For example, for some relative orientations of nucleons in the internal space [see Eq. (11)] the tensor part of the potential $V_{N N}^{T}$ makes a negative contribution to the total $N N$ potential. Such an attraction turned out to be sufficient for the construction of crystalline [8] or condensate [46] states of nuclear matter. Note, however, that the additional in-medium modifications of the $N N$ potential were not taken into account. In fact, previous Skyrme-model calculations [7] showed that the tensor part of the residual potential does decrease in the nuclear medium. Thus the Skyrme model allows us to combine the tensor and the central potentials into a full potential that should be shallow because of the medium influence. The question is whether the changes of the in-medium $N N$ potential are sufficient to induce a breakdown of the crystalline structure that does not seem to exist in nature. Of course, the medium modifications of the $N N$ potential might only be one of many agents that could induce this breakdown. For instance, quantum fluctuations $[46,47]$ and Fermi-motion effects should be of importance [48]. It is well known that the nuclear matter binding results from a strong cancellation of an attractive (binding) potential term and a repulsive kinetic term. It remains to be seen whether the condensed state of nuclear matter at ordinary densities can be achieved within the in-medium modified Skyrme model.

\section{ACKNOWLEDGMENTS}

This research is part of the EU Integrated Infrastructure Initiative Hadron Physics Project under contract number RII3CT-2004-506078 and is supported in part by DFG (SFB/TR 16) and by the Forschungszentrum Jülich under contract number 41445400 (COSY-067). U.T.Y. thanks A. K. Nasirov for useful correspondence and acknowledges support by Pusan National University (Post-Doc 2004 program).
[1] A. Jackson, A. D. Jackson, and V. Pasquier, Nucl. Phys. A432, 567 (1985).

[2] U. B. Kaulfuß and U.-G. Meißner, Phys. Rev. D 31, 3024 (1985).

[3] C. Hajduk and B. Schwesinger, Nucl. Phys. A453, 620 (1986).
[4] T. Otofuji, S. Saito, M. Yasuno, H. Kanada, and T. Kurihara, Prog. Theor. Phys. 78, 527 (1987).

[5] U. T. Yakhshiev, M. M. Musakhanov, A. M. Rakhimov, U.-G. Meißner, and A. Wirzba, Nucl. Phys. A700, 403 (2002). 
[6] U. T. Yakhshiev, U.-G. Meißner, and A. Wirzba, Eur. Phys. J. A 16, 569 (2003).

[7] A. M. Rakhimov, M. M. Musakhanov, F. C. Khanna, and U. T. Yakhshiev, Phys. Rev. C 58, 1738 (1998).

[8] I. R. Klebanov, Nucl. Phys. B262, 133 (1985).

[9] N. K. Glendenning, Phys. Rev. C 34, 1072 (1986).

[10] E. Wüst, G. E. Brown, and A. D. Jackson, Nucl. Phys. A468, 450 (1987).

[11] A. S. Goldhaber and N. S. Manton, Phys. Lett. B198, 231 (1987).

[12] M. Kugler and S. Shtrikman, Phys. Lett. B208, 491 (1988); Phys. Rev. D 40, 3421 (1989).

[13] A. D. Jackson and J. J. M. Verbaarschot, Nucl. Phys. A484, 419 (1988).

[14] L. Castillejo, P. S. J. Jones, A. D. Jackson, J. J. M. Verbaarschot, and A. Jackson, Nucl. Phys. A501, 801 (1989).

[15] H. Forkel, A. D. Jackson, M. Rho, C. Weiss, A. Wirzba, and H. Bang, Nucl. Phys. A504, 818 (1989).

[16] W. K. Baskerville, Nucl. Phys. A596, 611 (1996).

[17] N. S. Manton and P. J. Ruback, Phys. Lett. B181, 137 (1986).

[18] N. S. Manton, Commun. Math. Phys. 111, 469 (1987).

[19] A. D. Jackson, A. Wirzba, and L. Castillejo, Phys. Lett. B198, 315 (1987); Nucl. Phys. A486, 634 (1988); A. D. Jackson, C. Weiss, A. Wirzba, and A. Lande, ibid. A494, 523 (1989).

[20] A. D. Jackson, N. S. Manton, and A. Wirzba, Nucl. Phys. A495, 499 (1989).

[21] A. Wirzba and H. Bang, Nucl. Phys. A515, 571 (1990).

[22] A. D. Jackson, C. Weiss, and A. Wirzba, Nucl. Phys. A529, 741 (1991).

[23] A. Wirzba, in Baryons as Skyrme Solitons, edited by G. Holzwarth (World Scientific, Singapore, 1993), arXiv:hepph/9211295.

[24] B. D. Serot and J. D. Walecka, in Advances in Nuclear Physics, edited by J. W. Negele and E. Vogt (Plenum Press, New York, 1986), Vol. 16, p. 1.

[25] S. Gao, Y.-J. Zhang, and R.-K. Su, Nucl. Phys. A593, 362 (1995).

[26] Y.-J. Zhang, S. Gao, and R.-K. Su, Phys. Rev. C 56, 3336 (1997).

[27] L. Alvarez-Ruso, P. Fernandez de Cordoba, and E. Oset, Nucl. Phys. A606, 407 (1996).
[28] A. M. Rakhimov, U. T. Yakhshiev, and F. C. Khanna, Phys. Rev. C 61, 024907 (2000).

[29] U. T. Yakhshiev, A. W. Thomas, and F. C. Khanna, Phys. Rev. C 68, 048201 (2003).

[30] N. Kaiser and U.-G. Meißner, Phys. Lett. B233, 457 (1989).

[31] N. Kaiser and U.-G. Meißner, Nucl. Phys. A506, 417 (1990).

[32] M. M. Musakhanov and A. M. Rakhimov, Mod. Phys. Lett. A 10, 2297 (1995).

[33] H. Gomm, P. Jain, R. Johnson, and J. Schechter, Phys. Rev. D 33, 3476 (1986); A. A. Migdal and M. A. Shifman, Phys. Lett. B114, 445 (1982).

[34] V. A. Andrianov and V. Yu. Novozhilov, Phys. Lett. B202, 580 (1988).

[35] G. E. Brown and M. Rho, Phys. Rev. Lett. 66, 2720 (1991); M. Rho, Phys. Rep. 240, 1 (1994).

[36] G. E. Brown and M. Rho, Phys. Rep. 269, 333 (1996).

[37] M. M. Musakhanov, A. M. Rakhimov, U. T. Yakhshiev, and Z. Kanokov, Phys. Atom. Nucl. 62, 1845 (1999) [Yad. Fiz. 62, 1988 (1999)]; A. M. Rakhimov, F. C. Khanna, U. T. Yakhshiev, and M. M. Musakhanov, Nucl. Phys. A643, 383 (1998).

[38] T. E. Ericson and W. Weise, Pions and Nuclei (Clarendon, Oxford, 1988).

[39] A. M. Rakhimov, T. Okazaki, M. M. Musakhanov, and F. C. Khanna, Phys. Lett. B378, 12 (1996).

[40] E. M. Nyman and D.-O. Riska, Phys. Scripta 34, 533 (1986).

[41] A. I. Akhiezer, A. G. Sitenko, and V. K. Tartakovski, Nuclear Electrodynamics (Springer-Verlag, Berlin, 1994).

[42] U.-G. Meißner, Nucl. Phys. A503, 801 (1989).

[43] K. Saito, K. Tsushima, and A. W. Thomas, Phys. Rev. C 55, 2637 (1997).

[44] R.-K. Su, S.-J. Yang, S. Gao, and P. K. N. Yu, J. Phys. G 20, 1757 (1994).

[45] S. Gao, Y.-J. Zhang, and R.-K. Su, Phys. Rev. C 52, 380 (1995).

[46] D. I. D'yakonov and A. D. Mirlin, Sov. J. Nucl. Phys. 47, 421 (1988) [Yad. Fiz. 47, 662 (1988)].

[47] T. S. Walhout, Nucl. Phys. A484, 397 (1988); A519, 816 (1990).

[48] T. D. Cohen, Nucl. Phys. A495, 545 (1989). 